\title{
Instability of skyrmions in magnetic fields
}

Cite as: Appl. Phys. Lett. 116, 262406 (2020); https://doi.org/10.1063/5.0013488

Submitted: 12 May 2020 . Accepted: 16 June 2020. Published Online: 30 June 2020

Loic Mougel (D), Patrick M. Buhl (D), Ryohei Nemoto (D), Timofey Balashov (D), Marie Hervé, Julian Skolaut, Toyo Kazu Yamada (D), Bertrand Dupé (D), and Wulf Wulfhekel
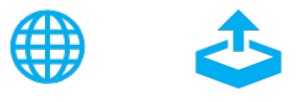

\section{Lock-in Amplifiers up to $600 \mathrm{MHz}$}
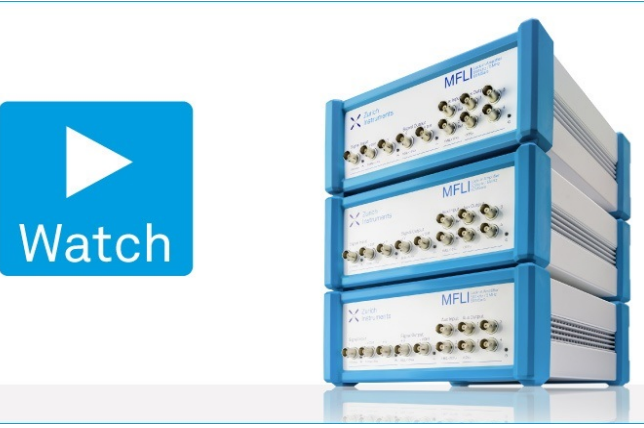


\title{
Instability of skyrmions in magnetic fields
}

\author{
Cite as: Appl. Phys. Lett. 116, 262406 (2020); doi: 10.1063/5.0013488 \\ Submitted: 12 May 2020 - Accepted: 16 June 2020 • \\ Published Online: 30 June 2020 \\ Loic Mougel, ${ }^{1, a)}$ iD Patrick M. Buhl, ${ }^{2}$ (iD Ryohei Nemoto, ${ }^{3}$ iD Timofey Balashov, ${ }^{7}$ (D Marie Hervé, ${ }^{1,4}$ Julian Skolaut, $^{7}$ \\ Toyo Kazu Yamada, ${ }^{3,5}$ (iD) Bertrand Dupé, ${ }^{6,7}$ (D) and Wulf Wulfhekel ${ }^{1}$ \\ AFFILIATIONS \\ ${ }^{7}$ Physikalisches Institut, Karlsruhe Institute of Technology, 76131 Karlsruhe, Germany \\ ${ }^{2}$ Institute of Physics, Johannes Gutenberg University Mainz, 55099 Mainz, Germany \\ ${ }^{3}$ Department of Materials Science, Chiba University, 1-33 Yayoi-cho, Inage-ku, Chiba 263-8522, Japan \\ ${ }^{4}$ Institut des Nanosciences de Paris, Sorbonne University, CNRS-UMR7588, 75005 Paris, France \\ ${ }^{5}$ Molecular Chirality Research Centre, Chiba University, 1-33 Yayoi-cho, Inage-ku, Chiba 263-8522, Japan \\ ${ }^{6}$ Fonds de la Recherche Scientifique (FNRS), B-1000 Bruxelles, Belgium \\ ${ }^{7}$ Nanomat/Q-mat/CESAM, Université de Liège, B-4000 Sart Tilman, Belgium
}

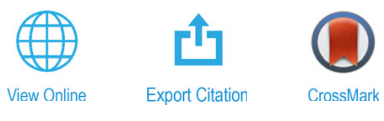

${ }^{\text {a) }}$ Author to whom correspondence should be addressed: loic.mougel@kit.edu

\begin{abstract}
In this combined experimental and theoretical work, we report on the evolution of the skyrmion radius and its destruction in the system $\mathrm{Co} / \mathrm{Ru}(0001)$ when an out-of-plane magnetic field is applied. At low fields, skyrmions are metastable and display an elliptical instability in which along the short axis, the spin texture approaches that of the spin-spiral phase and the long axis expands in order to go back to the spin-spiral ground state. At high fields, we observe round skyrmions of finite size up to the collapse field $B_{c}$, where they are destroyed and the topological charge is annihilated. We estimate $B_{c}$ via numerical methods based on magnetization dynamics simulations parametrized by density functional theory calculations and compare it to experimental scanning tunneling microscopy observations obtained at $\approx 30 \mathrm{mK}$.
\end{abstract}

Published under license by AIP Publishing. https://doi.org/10.1063/5.0013488

Magnetic skyrmions are localized topologically protected structures, in which the magnetization field winds around a magnetization core, such that no continuous transformation allows the conversion into a topologically trivial, homogeneously magnetized ferromagnetic (FM) state. ${ }^{1-3}$ The energetic stability of the skyrmions originates from the unique sense of rotation of the magnetization due to the chiral Dzyaloshinskii-Moriya interaction (DMI) which occurs when structural inversion symmetry is broken and spin-orbit coupling is present. ${ }^{4}$ Due to this protection, skyrmions are considered as magnetic quasiparticles and are discussed as ideal information carriers in spintronics. $^{5-12}$

When a magnetic field is applied in the opposite direction to the magnetic moment of the skyrmion center, the Zeeman energy leads to a compression of the skyrmion. ${ }^{13-15}$ When the magnetic field reaches a critical value $B_{c}$, the core of the skyrmion will reverse its magnetization and align to the FM background. This mechanism is called the collapse of the skyrmion. In the continuum limit, the collapse of skyrmions occurs when their radii shrink down to zero, ${ }^{16}$ while on a finite lattice of magnetic atoms, the radius reaches a finite value before the collapse. ${ }^{14,16}$ Skyrmion collapse with its abrupt change of the topology is associated with an energetic barrier and is usually studied as being thermally activated within the framework of transition state theory. ${ }^{17}$

Transition state theory requires the exploration of high energy states, the so-called saddle points, via the nudged elastic band (NEB). ${ }^{18}$ In magnetism, NEB has been extended to take into account the constant size of the magnetic moments and is known as the geodesic nudged elastic band (GNEB). ${ }^{19,20}$ This method has been used extensively to study the thermally activated probability of skyrmion collapse in strong ferromagnets such as $\mathrm{Co} / \mathrm{Pt}(111)^{14}$ and in ultrathin magnetic films such as $\mathrm{Pd} / \mathrm{Fe} / \mathrm{Ir}(111){ }^{21-23}$ Recently, more approaches for thermally activated skyrmion collapse have been proposed via Langevin magnetization dynamics simulation. ${ }^{24}$

Although the thermally activated transition of single skyrmions to a ferromagnetic state has been extensively studied, the exploration of the transition by ramping up the external magnetic field at low temperatures-which involves the exploration of the saddle pointremains unexplored. This low temperature transition is particularly important in spin-polarized STM experiments which are done at cryogenic temperatures on ultra-thin films. ${ }^{25,26} \mathrm{In} \mathrm{Pd} / \mathrm{Fe} / \mathrm{Ir}(111)$, the transition between the skyrmion lattice phase and the FM phase as a 
function of the external magnetic field has been measured at $4 \mathrm{~K}^{13,25}$ The skyrmion radius decreases as the magnetic field increases. However, in $\mathrm{Pd} / \mathrm{Fe} / \mathrm{Ir}(111)$, the large value of $B_{c}$ did not allow the exploration of the saddle point. ${ }^{13}$

Compared to the experiment, the saddle point configuration is easy to explore in calculations since the magnetic configuration can be constrained. At this point, the magnetic moments are expected to align in-plane to create a vortex whose size is only several unit cells. ${ }^{20}$ This vortex unwinds the skyrmion and cancels the topological stabilization. Recently, the saddle point configurations were explored theoretically, and it was found that even when the magnetic interactions are described in the continuum limit, a skyrmion of finite size could be expected as the saddle point configuration. ${ }^{27}$

Here, we study the skyrmion collapse field $B_{c}$ of isolated skyrmions in Co islands of single atomic thickness on $\mathrm{Ru}(0001)$. In a previous work, ${ }^{26}$ we have combined spin-polarized STM measurements and DFT calculations to explore the ground state properties of $\mathrm{Co} / \mathrm{Ru}(0001)$ and showed that skyrmions were stabilized by a vanishing effective anisotropy in combination with a small DMI. This particular ratio of magnetic interactions makes $\mathrm{Co} / \mathrm{Ru}(0001)$ a prototypical soft ferromagnet in which isolated skyrmions have been stabilized at down to zero external magnetic fields. We experimentally determine $B_{c}$ for skyrmion annihilation at minimal thermal activation using a dilution refrigerated STM with $T \approx 30 \mathrm{mK}^{28}$ We numerically estimate $B_{c}$ based on $a b$ initio parameters and find that it agrees well with experimental observations.

The ground state of $\mathrm{Co} / \mathrm{Ru}(0001)$ in zero external magnetic field is a spin spiral of unique rotational sense and a periodicity of $37 \mathrm{~nm} .{ }^{26}$ When a magnetic field $B$ is applied normal to the surface, the spin spiral successively transforms into isolated skyrmions with radii depending on the external magnetic field. In our previous work, the spin structures were studied using tunneling anisotropic magnetoresistance (TAMR) and tunneling magneto-resistance (TMR). Here, we exclusively used the TAMR signal to determine the shape of the skyrmions to avoid magnetic dipole interactions between the tip and the sample and tracked the skyrmion dimensions as a function of magnetic field as shown Fig. 1. Note that in these maps of the differential conductivity $d I / d V$ recorded at $-350 \mathrm{mV}$, areas of an in-plane magnetization appear bright (higher $d I / d V$ ) opposed to areas with an out-of-plane magnetization appearing black (lower $d I / d V$ ). ${ }^{26,29,30}$ Although the Ru substrate becomes superconducting below a critical temperature of $\approx 500 \mathrm{mK}$, all measurements presented were obtained in magnetic fields above the superconducting upper critical field of $\approx 5 \mathrm{mT}$, i.e., in the normal state of the substrate. ${ }^{31}$ In our experiments, skyrmions were formed by first applying $250 \mathrm{mT}$. Then, the $B$ was reduced to the remanence of the superconducting magnet $(\approx 7 \mathrm{mT})$ and metastable skyrmions remained. After this formation step, $B$ was increased again to the indicated magnitudes to study the evolution of the skyrmion dimensions as function of $B$.

For fields below $B_{e}=230 \mathrm{mT}$, the skyrmions are found to be not round but elongated as seen in the left inset of Fig. 1. This is due to their tendency to re-form the spin-spiral state at low fields via an instability against elongation. Thus, we determined the largest and smallest axis of the elongated skyrmion indicated in orange and green lines in the left inset and open circles in the figure. At fields higher than $230 \mathrm{mT}$, the skyrmions are round objects (see right inset of Fig. 1). The $d I / d V$ signal varies as $\cos ^{2}(\theta)$, with $\theta$ being the azimutal angle. The function used to fit the radius of the skyrmion is ${ }^{32}$

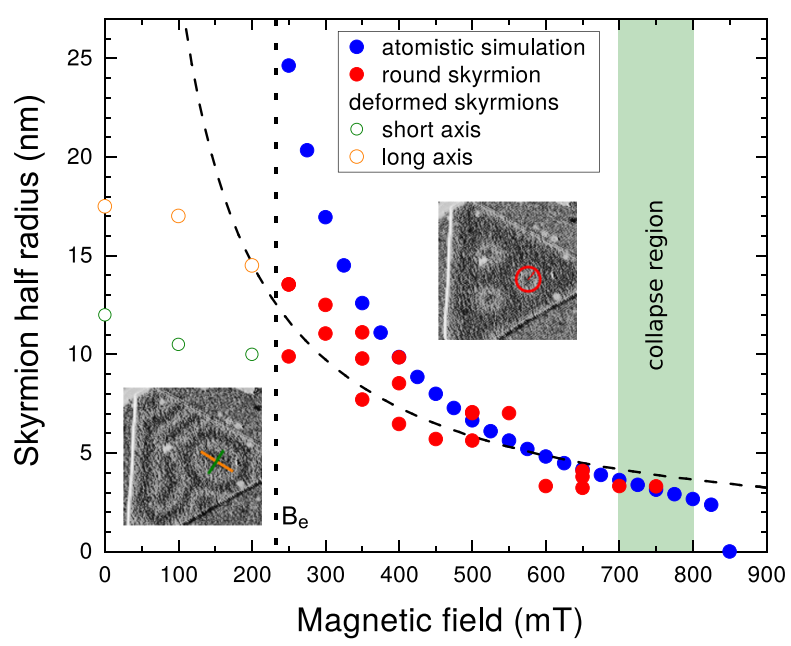

FIG. 1. Theoretical (blue filled circles) values of $\sigma / 2$ of the skyrmion vs applied magnetic field in $\mathrm{Co} / \mathrm{Ru}(0001)$ together with the experimental values for round skyrmions $B>B_{e} \approx 230 \mathrm{mT}$ as illustrated in the right inset. At $B<B_{e}$, the skyrmions are elongated, and both the radii along the long (orange) and short (green) axis are plotted as illustrated in the left inset. The black dashed curve is a fit to a/B. At fields in the shaded green area, annihilation of skyrmions was experimentally observed. The insets are differential conductance maps (white level proportional to $d / l d V$ ) of $130 \times 130 \mathrm{~nm}, V=-350 \mathrm{mV}, I=2 \mathrm{nA}$, modulation: $U=60 \mathrm{mV}$. Left inset: $B=0 \mathrm{mT}$ and right inset: $B=400 \mathrm{mT}$.

$$
d I / d V= \begin{cases}\cos ^{2}\left(\pi \frac{\rho}{\sigma}\right) & \text { if } \rho \leq \sigma \\ 0 & \text { otherwise }\end{cases}
$$

where $\rho$ is the distance from the center of the skyrmion and $\sigma$ is the radius of the skyrmion. The distance from the center of the skyrmion to the maximum brightness in the $d I / d V$ maps, thus, corresponds to half the skyrmion radius. We fitted the $d I / d V$ contrast as shown in the right inset of Fig. 1 via Eq. (1) to obtain the skyrmion radii for different magnetic fields. The radii of the round skyrmions as a function of the magnetic field are shown as red dots in Fig. 1. At lower fields, the scatter in the data is explained by the mentioned elliptical instability and the skyrmions' sensitivity to the near spin-spiral phase. As the field increases, these effects become marginal and the radius of the different skyrmions converges to the same value.

At higher field, analytical models predict the radius of isolated skyrmions to scale as $1 / B^{33,34}$ as indicated by a fit to the high field experimental data (black dashed line). At fields below $B_{e}$, this relation necessarily breaks down as the ground state is not a single, infinitely extended skyrmion ${ }^{35}$ but the spin-spiral phase. Note that for the elongated skyrmions, the short axis half-radius saturates around $12 \mathrm{~nm}$, corresponding to a local periodicity of the spin-spiral of $\approx 48 \mathrm{~nm}$, i.e., at similar dimensions as the periodicity of the spin spiral. ${ }^{26}$ The long axis expands further in order to transform into the spin-spiral phase. Due to the neighboring spin spirals in the sample and the finite sample size, the long axis, however, does not diverge to infinity. In other words, a kinetic barrier to go back to the spin-spiral phase allows for metastable skyrmions at vanishing fields. At fields larger than $B_{e}$, the round skyrmions are compressed and the radii keep decreasing but stay finite until $B=700 \mathrm{mT}$. At this field, the collapse region is 
reached and individual skyrmions disappear by annihilating the topological charge (and do not reappear upon lowering the field again). When the field reaches $B=800 \mathrm{mT}$, all skyrmions disappear which shows that the collapse field $B_{c}$ was reached at $B_{c}=750 \pm 50 \mathrm{mT}$. As all measurements are made at $T \approx 30 \mathrm{mK}$, this collapse is not due to thermal activation. Moreover, during ramping the field, the tip was retracted such that an influence of the tunneling current on the skyrmion collapse was excluded.

The experimental measurements were compared with atomistic simulations utilizing the Spirit code ${ }^{36}$ which describes the energy of the magnetic texture as

$$
\begin{aligned}
E= & -\sum_{\mathrm{ij}} J_{\mathrm{ij}} \mathbf{M}_{\mathrm{i}} \mathbf{M}_{\mathrm{j}}-\sum_{\mathrm{ij}} \mathbf{D}_{\mathrm{ij}}\left(\mathbf{M}_{\mathrm{i}} \times \mathbf{M}_{\mathrm{j}}\right) \\
& -\sum_{\mathrm{i}} M_{s} \mathbf{M}_{\mathrm{i}} \mathbf{B}-\sum_{\mathrm{i}} \kappa \mathbf{M}_{\mathrm{i}, \mathrm{z}}^{2} \\
& -\sum_{\mathrm{ij}} \frac{\mu_{0} M_{s}^{2}}{4 \pi} \frac{\mathbf{M}_{\mathrm{i}} \mathbf{M}_{\mathrm{j}} r_{\mathrm{ij}}^{2}-\left(\mathbf{M}_{\mathrm{i}} \mathbf{r}_{\mathrm{ij}}\right)\left(\mathbf{M}_{\mathrm{j}} \mathbf{r}_{\mathrm{ij}}\right)}{r_{\mathrm{ij}}^{5}},
\end{aligned}
$$

where $J_{\mathrm{ij}}$ is the magnetic exchange interaction, $\mathbf{D}_{\mathrm{ij}}$ is the Dzyaloshinskii-Moriya vector, $\kappa$ is the magnetocrystalline anisotropy (MAE), and $\mathbf{B}$ is the external magnetic field. The magnetic interaction parameters, obtained by DFT in a previous publication, ${ }^{26}$ are $J_{1}=13.1 \mathrm{meV}, D_{1}=0.2 \mathrm{meV}, \kappa=-0.013 \mathrm{meV}$, and $M_{s}=1.8 \mu_{\mathrm{B}}$. Using these parameters, the experimental setup was modeled by a periodic superlattice containing $1000 \times 1000$ magnetic moments on a hexagonal lattice with the Ru lattice parameter of $a=0.27 \mathrm{~nm}$. For each magnetic field $H$, an isolated skyrmion was relaxed to its minimum of energy at which the skyrmion radius was fitted with Eq. (1).

As in Ref. 30, the radius of the skyrmion without the dipoledipole interaction (DDI) was calculated as a function of the field (blue dots in Fig. 1). Compared to the experiment, the radius decreases more steeply with increasing field but follows the same trend and matches well at higher fields. This discrepancy at lower fields is to be expected as the experimental skyrmions are more densely packed, which leads to additional skyrmion compression compared to the simulations. The atomistic simulations predict a collapse field of $B_{c}$ $=820 \pm 10 \mathrm{mT}$ which is in good agreement with the experimental value. Considering that all theoretical parameters were obtained from DFT, the agreement between the atomistic simulations and the experiments is extremely good. This is particularly remarkable as tiny modifications of the anisotropy on the scale of the DFT-accuracy result in drastic modifications of the skyrmion radii and $B_{c}$ in this fragile regime of atomistic parameters.

Additionally including the often neglected DDI demonstrates the delicate balance of atomistic parameters in this soft magnet. First, the DDI was evaluated in a magnetic configuration that was minimized neglecting it [Figs. 2(a), 2(b), 2(d), and 2(e)]. The energy gain of the skyrmion compared to the ferromagnetic state through the DDI is more than 5 times the gain of the MAE. Nevertheless, qualitatively both distributions do not differ much except for the non-vanishing energy gain at the core in the case of the DDI. This is consistent with the common 2D interpretation of the DDI as shape anisotropy which can be absorbed into an effective magnetic anisotropy. ${ }^{37}$ Due to the relatively large magnitude of the DDI in this fragile system, the magnetic ground state changes drastically when the DDI is included. The background magnetization tilts in-plane in the experimentally
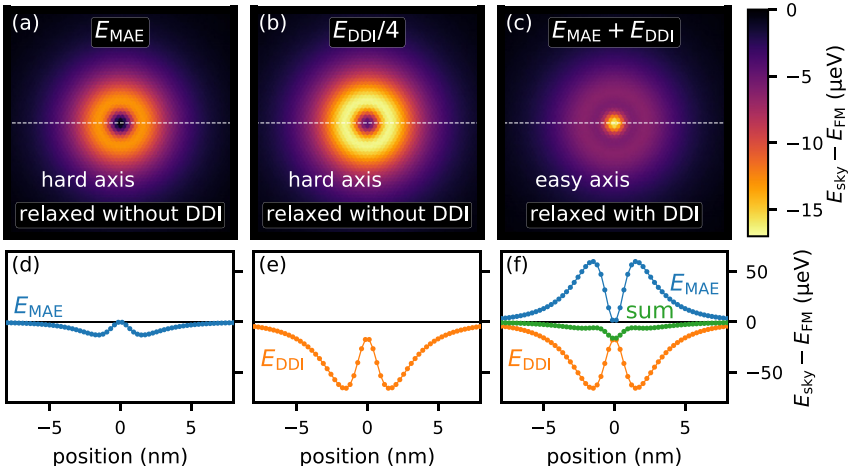

FIG. 2. Energy density distribution with respect to the FM state at $B=750 \mathrm{mT}$. (a) and (b): The skyrmion was relaxed with an easy axis in-plane anisotropy of $13 \mu \mathrm{eV}$ and without DDI: (a) MAE contribution and (b) DDI contribution. (c) Skyrmion relaxed with both $\mathrm{DDI}$ and an easy axis anisotropy out-of-plane of $60 \mu \mathrm{eV}$. (d)-(f) Radial energy density distribution showing the MAE (d), the DDI (e) and both contribution ( $\mathrm{f}$. The integral of (a) and (c) is of comparable magnitude with $13.8 \mathrm{meV}$ and $11.8 \mathrm{meV}$, respectively.

investigated field range and skyrmions in an out-of-plane background are only viable in a small region around $1.5 \mathrm{~T}$. In order to regain the previous agreement with experiment, the MAE as the smallest parameter with large theoretical uncertainty and similar impact was tuned to $\kappa=0.06 \mathrm{meV}$, which approximately counters the energy gain through the DDI. While the shape of the energy gain from the effective anisotropy shifts toward the core when including the DDI and modifying $\kappa$ [Figs. 2(c) and 2(f)], the previous dependence of the skyrmion radius on the field is recovered (not shown). Hence, the experimental results can also be described when including DDI although a reasonable but arbitrary modification to the MAE is necessary.

Since DDI is not required to study the stability of skyrmions in ultra-thin films, we have explored the energy barrier without explicitly considering DDI in the vicinity of $B_{c}$ via GNEB simulations ${ }^{19}$ on a $400 \times 400$ grid as shown in Fig. 3 . The simulations were initialized with the relaxed skyrmion profile for a given magnetic field. Along the reaction path, the skyrmion shrinks until it reaches the saddle point

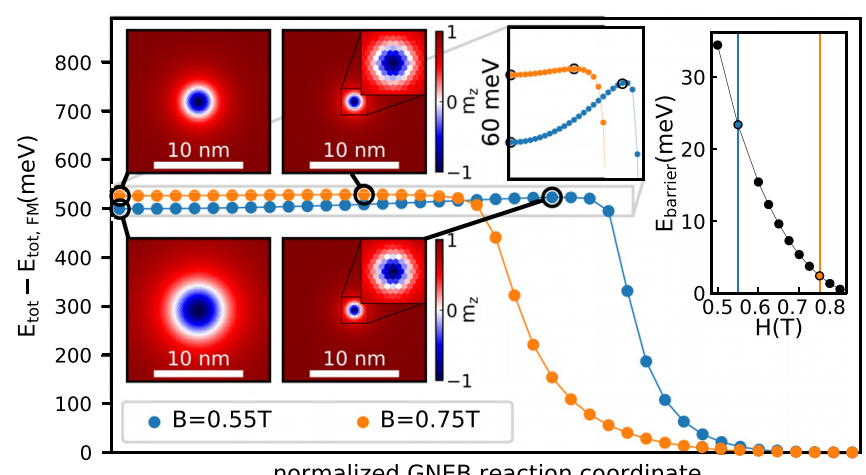

FIG. 3. GNEB calculation for $B=0.55 \mathrm{mT}$ (blue) and $B=0.75 \mathrm{mT}$ (orange) with zoom-in between 485 and $545 \mathrm{meV}$. The right inset shows the magnetic field dependence of the GNEB energy barrier. The four left insets depict the distributions of the z-component of the magnetization including zooms of the saddle-point core. 
which corresponds to the maximum of energy. The energy maximizes as the half-radius reaches about $2 \mathrm{~nm}$ after which the core reverses and the ferromagnetic state is quickly attained (toward the right side of the figure). In that respect, even at the saddle point, we confirm numerically that in soft ferromagnets a finite radius skyrmion solution exists as predicted in Ref. 27.

When the external magnetic field is increased, the energy barrier decreases drastically (right inset of Fig. 3), reaching $0.5 \mathrm{meV}$ at $800 \mathrm{mT}$. For all the magnetic fields, the skyrmion solution at the saddle point has a finite radius. The initial flat section of the energy evolution easily allows variation of the skyrmion radius in the vicinity of $B_{c}$, while the very small barrier at $B_{c}$ prohibits direct observation of the skyrmion collapse. Still, with the minimal experimental skyrmion halfradius of $\approx 3.1 \mathrm{~nm}$, the direct vicinity of the collapse is observed to be a good approximation, and all further compression leads to vanishing of the barrier (or tunneling) on time scales too small for observation.

To conclude, the collapse path of a skyrmion was studied in the prototypical soft ferromagnet $\mathrm{Co} / \mathrm{Ru}(0001)$. As expected, the radius of the skyrmion decreases monotonically as the magnetic field increases up to the collapse field $B_{c}$. We have reproduced the experimental skyrmion radii and computed $B_{c}$ based on atomistic simulation parameterized by DFT calculation. Both experimental and computational methods confirm that its radius does not reach zero even at the saddle point and that the DDI can be correctly approximated by an effective anisotropy in $2 \mathrm{D}$ ultra-thin films.

B. Dupé and P. Buhl acknowledge useful discussion with J. V. Kim and U. Ritzmann. W. Wulfhekel and L. Mougel acknowledge funding by the Deutsche Forschungsgemeinschaft (DFG) under Grant Nos. WU 349/15-1 and WU 349/16-1. B. Dupé and P. Buhl acknowledge funding by the DFG under Grant No. DU 1489/3-1.

\section{DATA AVAILABILITY}

The data that support the findings of this study are available from the corresponding author upon reasonable request.

\section{REFERENCES}

${ }^{1}$ A. N. Bogdanov and A. Hubert, Phys. Status Solidi 186, 527 (1994).

${ }^{2}$ U. K. Rössler, A. N. Bogdanov, and C. Pfleiderer, Nature 442, 797 (2006).

${ }^{3}$ A. N. Bogdanov and D. Yablonskii, Zh. Eksp. Teor. Fiz. 95, 178 (1989).

${ }^{4}$ A. Fert and P. M. Levy, Phys. Rev. Lett. 44, 1538 (1980).

${ }^{5}$ N. S. Kiselev, A. N. Bogdanov, R. Schäfer, and U. K. R. Ler, J. Phys. D 44, 392001 (2011).

${ }^{6}$ F. Jonietz, S. Mühlbauer, C. Pfleiderer, A. Neubauer, W. Münzer, A. Bauer, T. Adams, R. Georgii, P. Böni, R. A. Duine, K. Everschor, M. Garst, and A. Rosch, Science 330, 1648 (2010).
${ }^{7}$ A. Fert, V. Cros, and J. Sampaio, Nat. Nanotechnol. 8, 152 (2013).

${ }^{8}$ J. Sampaio, V. Cros, S. Rohart, A. Thiaville, and A. Fert, Nat. Nanotechnol. 8, 839 (2013).

${ }^{9}$ N. Nagaosal and Y. Tokura, Nat. Nanotechnol. 8, 889 (2013).

${ }^{10}$ A. Fert, N. Reyren, and V. Cros, Nat. Rev. Mater. 2, 17031 (2017).

${ }^{11}$ T. Nozaki, Y. Jibiki, M. Goto, E. Tamura, T. Nozaki, H. Kubota, A. Fukushima,

S. Yuasa, and Y. Suzuki, Appl. Phys. Lett. 114, 012402 (2019).

${ }^{12}$ C. Ma, X. Zhang, J. Xia, M. Ezawa, W. Jiang, T. Ono, S. N. Piramanayagam, A. Morisako, Y. Zhou, and X. Liu, Nano Lett. 19, 353 (2019).

${ }^{13} \mathrm{~N}$. Romming, A. Kubetzka, C. Hanneken, K. von Bergmann, and R. Wiesendanger, Phys. Rev. Lett. 114, 177203 (2015).

${ }^{14}$ S. Rohart, J. Miltat, and A. Thiaville, Phys. Rev. B 93, 214412 (2016).

${ }^{15}$ A. Bernand-Mantel, L. Camosi, A. Wartelle, N. Rougemaille, M. Darques, and L. Ranno, SciPost Phys. 4, 027 (2018).

${ }^{16}$ A. Leonov, T. L. Monchesky, N. Romming, A. Kubetzka, A. N. Bogdanov, and R. Wiesendanger, New J. Phys. 18, 065003 (2016).

${ }^{17}$ E. Wigner, Trans. Faraday Soc. 34, 29 (1938).

${ }^{18}$ G. Henkelman, B. P. Uberuaga, and H. Jónsson, J. Chem. Phys. 113, 9901 (2000).

${ }^{19}$ P. F. Bessarab, V. M. Uzdin, and H. Jónsson, Phys. Rev. B 85, 184409 (2012).

${ }^{20}$ P. F. Bessarab, V. M. Uzdin, and H. Jónsson, Comput. Phys. Commun. 196, 335 (2015).

${ }^{21}$ S. von Malottki, B. Dupé, P. F. Bessarab, A. Delin, and S. Heinze, Sci. Rep. 7, 12299 (2017).

${ }^{22}$ P. F. Bessarab, G. P. Müller, I. S. Lobanov, F. N. Rybakov, N. S. Kiselev, H. Jónsson, V. M. Uzdin, S. Blügel, L. Bergqvist, and A. Delin, Sci. Rep. 8, 3433 (2018).

${ }^{23}$ S. von Malottki, P. F. Bessarab, S. Haldar, A. Delin, and S. Heinze, Phys. Rev. B 99, 060409 (2019).

${ }^{24}$ L. Desplat, C. Vogler, J.-V. Kim, R. L. Stamps, and D. Suess, Phys. Rev. B 101, 060403 (2020).

${ }^{25} \mathrm{~N}$. Romming, C. Hanneken, M. Menzel, J. E. Bickel, B. Wolter, K. von Bergmann, A. Kubetzka, and R. Wiesendanger, Science 341, 636 (2013).

${ }^{26}$ M. Hervé, B. Dupé, R. Lopes, M. Böttcher, M. D. Martins, T. Balashov, L. Gerhard, J. Sinova, and W. Wulfhekel, Nat. Commun. 9, 1015 (2018).

${ }^{27}$ B. Heil, A. Rosch, and J. Masell, Phys. Rev. B 100, 134424 (2019).

${ }^{28}$ T. Balashov, M. Meyer, and W. Wulfhekel, Rev. Sci. Instrum. 89, 113707 (2018).

${ }^{29}$ M. Bode, S. Heinze, A. Kubetzka, O. Pietzsch, X. Nie, G. Bihlmayer, S. Blügel, and R. Wiesendanger, Phys. Rev. Lett. 89, 237205 (2002).

${ }^{30} \mathrm{M}$. Hervé, T. Balashov, A. Ernst, and W. Wulfhekel, Phys. Rev. B 97, 220406 (2018).

${ }^{31}$ D. K. Finnemore and D. E. Mapother, Phys. Rev. Lett. 9, 288 (1962).

${ }^{32}$ K. von Bergmann, M. Menzel, D. Serrate, Y. Yoshida, S. Schröder, P. Ferriani, A. Kubetzka, R. Wiesendanger, and S. Heinze, Phys. Rev. B 86, 134422 (2012).

${ }^{33}$ A. N. Bogdanov and A. Hubert, J. Magn. Magn. Mater. 138, 255 (1994).

${ }^{34}$ M. N. Wilson, A. B. Butenko, A. N. Bogdanov, and T. L. Monchesky, Phys. Rev. B 89, 094411 (2014).

${ }^{35}$ A. N. Bogdanov and A. Hubert, J. Magn. Magn. Mater. 195, 182 (1999).

${ }^{36}$ G. P. Müller, M. Hoffmann, C. Dißelkamp, D. Schürhoff, S. Mavros, M. Sallermann, N. S. Kiselev, H. Jónsson, and S. Blügel, Phys. Rev. B 99, 224414 (2019).

${ }^{37}$ E. Feldtkeller and H. Thomas, Phys. Kondens. Mater. 4, 8 (1965). 